\title{
ANÁLISIS DE LA ACTITUD DE INNOVACIÓN TEGNOLÓGICA EN EL PERÚ: EL CASO DE LOS MEDIOS DE COMUNICACIÓN ELECTRÓNICA
}

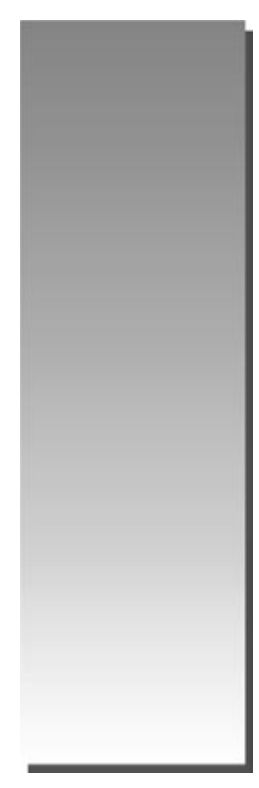

Augusto Hidalgo Sánchez* E-mail: ahidalgos@unmsm.edu.pe

\begin{abstract}
RESUMEN
La innovación tecnológica en el Perú ocurre al azar. Históricamente, los actores del desarrollo económico y social del país parecen no ser conscientes de la importancia de la adopción de tecnología. Actúan concentrados en la política económica o en los beneficios a obtener, y descuidan su impacto sobre el desarrollo. Esto se ve confirmado por esta breve narración sobre la incorporación de tecnología de medios electrónicos en el Perú.
\end{abstract}

Palabras clave: Telefonía Perú, celulares, teléfonos fijos, innovación tecnológica.

\section{ABSTRACT}

Technological innovation in Peru happens randomly. Historically, the actors for the economic and social development of the country seem not to be aware of the importance of technology adoption. They behave concentrated on the economic or political profits, and carelessly regarding its impact on development. This is confirmed by this short narration about the incorporation of electronic media technology in Peru.

Key words: Phone system Peru, mobill, telephones, technological innovation.

* Master of Science y MBA. Licenciado en Administración. Profesor de la Facultad de Ciencias Administrativas (UNMSM). Consultor en asuntos de comunicaciones y tecnologías de información. 


\section{INTRODUCCIÓN}

La actual euforia económica que se está viviendo en el Perú ha causado la súbita ilusión de que pronto seremos un país desarrollado. Esto es bueno ya que la ciencia de la Economía nos enseña que la confianza en ella y la ilusión de una vida mejor es un gran acelerador de la producción, el consumo y la innovación, pero no hay que ir tan de prisa. Si bien es cierto que el Producto Bruto Interno (PBI) per cápita (IPC) de los peruanos se ha duplicado en los últimos cinco años, llegando a los US\$ 4,000 nominales, esta cifra, en términos del poder de compra efectivo o purchasing-power-parity (PPP), arroja US\$ 7,803 por persona de acuerdo al cálculo del Fondo Monetario Internacional (FMI), lo que a su vez ubica al país en el puesto 84 del IPC a nivel mundial, por encima de otros países latinoamericanos como Colombia o Ecuador y también arriba de algunos países de Europa como Ucrania, o de Asia como la misma China, pero nuestro país está muy lejos de los países desarrollados, algunos de los cuales superan los US\$ 80,000 en PPP.

Ciertamente que el camino al desarrollo en estos tiempos modernos del siglo XXI no es imposible; las experiencias de países como Chile, Brasil y la República Popular China son inspiradoras pero, para encontrar el camino, se necesita hacer muy bien las cosas y probablemente, en unos treinta años, podríamos unirnos a ese club.

Pero ¿qué significa hacer bien las cosas? Significa, por ejemplo, incorporar masivamente la tecnología para la producción privada y pública de bienes así como para la prestación de servicios básicos como educación, salud, transporte, justicia, defensa, etc. Y, aquí es precisamente donde el Perú ha dado hasta el momento muestras poco contundentes de poseer una vocación de innovación para la mejora de la productividad. Más bien las muestras están llenas de indiferencia y de poco cariño por la innovación tecnológica, tanto en el sector privado como en el público. Nuestros actores sociales, sean los políticos, los gobernantes, los empresarios, los académicos, los comunicadores o los simples ciudadanos, parecen no comprender o no intuir el impacto de la tecnología en la competitividad del país. Parece que la tecnología es tomada con cierta liviandad. Voy a dar algunas pruebas de lo que afirmo, solamente a partir del caso de la incorporación de la tecnología de comunicación electrónica en el Perú durante los últimos cien años.
BREVE RELATO DE LAINNOVACIÓN TECNOLÓGICA EN LAS COMUNICACIONES ELECTRÓNICAS EN EL PERÚ

Veamos sucintamente lo que los actores del desarrollo económico y social han hecho en el Perú durante las experiencias de introducción de la radio, de la televisión, de los satélites, de la cámara filmadora, de la telefonía, y de la computadora e Internet.

\section{La Radio}

En 1908 Guglielmo Marconi patentó la radiocomunicación (no hablada) de señales Morse, es decir la telegrafía sin hilos, o el telegrama radial, que luego dio nacimiento a la radio. Dos años más tarde el gobierno de Estados Unidos aprobó un acta por la cual se obligaba a toda embarcación mayor de cincuenta ocupantes a instalar uno de estos aparatos como condición indispensable para poder hacerse a la mar. En 1912 se hundió el Titanic y de no haber sido por este invento seguramente se hubieran ahogado unas 5000 personas y no las 1200 que perecieron. Un siglo después, en nuestro país siguen perdiéndose lanchas con pescadores artesanales, como si desde entonces no se hubieran registrado en el mundo unas 6000 patentes al respecto.

Pero no se crea que el Perú estuvo ajeno o ignorante a las noticias de los inventos de la radio. Al contrario, su entusiasmo fue desbordante. Está registrado en la historia del Perú que en el año 1903 acoderó en el Callao el crucero Elba de la Real Armada Italiana. Este barco probó en Lima un extraordinario aparato llamado radio. Los limeños se quedaron asombrados. Nueve años más tarde, en 1912, el gobierno del presidente Augusto B. Leguía inauguró la primera estación radiotelegráfica de Lima en el cerro San Cristóbal. Es decir, fue creada la primera empresa pública radial de comunicación telegráfica pero, a diferencia del caso norteamericano, no se conoce que se sancionara norma alguna para su uso masivo en el transporte o en la industria del Perú.

Posteriormente, en 1920, nació oficialmente la radiocomunicación comercial sonora o simplemente la radio, con la salida al aire de la estación KDKA de Pittsburgh, Estados Unidos. En el Perú, en 1921, don Jorge Vargas Escalante estableció conexión directa con la Estación Darién de Panamá. Luego, en 1925 la empresa privada Compañía Peruana de Broadcasting, con apoyo tecnológico 
y financiero de la Marconi Wireless Telegraph Co. Ltd. de Inglaterra, creó la estación OAX, la primera radio peruana, que más tarde se convirtiría en Radio Nacional. El 20 de junio de ese año se instalaron en la Plaza de Armas de Lima receptores especiales para la escucha de la gente allí congregada. En esa ocasión el Presidente Leguía inauguró la estación con estas esperanzadoras frases: "la inauguración de este servicio de radiodifusión es la prueba más concluyente de que el progreso del Perú marcha a grandes pasos hacia un porvenir que por ahora no podemos ni siquiera vislumbrar"1.

La radio se convirtió en Lima en la distracción nocturna de moda para quienes tenían el poder económico de adquirir un receptor. El primer director artístico de la OAX fue don Antonio Garland, sus primeros locutores el señor Juan Fernández y la señora Rosa Hernández.

La Radio OAX, como novedad, fue éxito rotundo, pero como empresa resultó en un sonoro fracaso: la Marconi no llegó a vender suficientes equipos receptores, lo cual era su objetivo. Ante esa circunstancia el Estado peruano la adquirió por considerar que era de necesidad pública y tal vez pensando en su aprovechamiento político. En 1926 la OAX pasó a la Dirección de Correos del Ministerio de Gobierno y Policía. Con el financiamiento del presupuesto público suficiente, la OAX dedicó su programación a conciertos, sermones, conferencias, eventos deportivos, etc. En 1933, cambió su nombre por Radio Nacional del Perú.

A partir de 1934, y luego que una masa crítica de la población limeña había adquirido sus receptores, nacen las radios privadas Grellaud, Excelsior, Flores y Goicochea, cuyo objetivo era vender publicidad. En 1936 se lanza por primera vez un informativo noticioso y ese mismo año esta nueva tecnología es utilizada en la campaña política de ese entonces. En 1968 ya existían 222 emisoras, cinco de ellas estatales, 37 en Lima y 185 en el resto del país.

La radiodifusión peruana nació bajo el modelo norteamericano de empresa privada autofinanciada. A este rasgo se le agregó el hecho que, para los efectos prácticos, nunca se le impuso regulación alguna que tuviera relación con los objetivos del país, ni siquiera con la calidad de los programas emitidos. El entretenimiento y la información fue el principal giro de las radios, giro que probó ser efectivo para atraer audiencias sufi- cientes para, a su vez, atraer publicidad y ventas. Esa es la lógica de la radiodifusión comercial.

\section{La Televisión}

En 1939 se presentó en sociedad la televisión, pero recién en 1944 fue lanzada como producto comercial y es a partir de 1946 que se masifica en los hogares norteamericanos, dando un salto gigantesco e irreversible.

En el Perú la televisión, a diferencia de la radio, llegó un poco retrasada, eso sí con fines culturales. Precisamente, el 17 de Enero de 1958 el Ministerio de Educación, en convenio con la Organización de las Naciones Unidas para la Educación, la Ciencia y la Cultura (UNESCO), lanzó al aire desde el último piso de su edificio en la Avenida Abancay de Lima, un modesto canal educativo que sirviera a su vez de entretenimiento. La inversión fue de US\$ 22,000 en equipos para este Canal 5 , pero no fue suficiente como para construir la estructura de un medio dedicado a la cultura, por esa razón pronto entró en crisis.

Los empresarios privados radiales, agrupados en la Junta Permanente Nacional de Telecomunicaciones, lograron que el Canal 5 pasara a manos de inversionistas y para el Estado se reservó la señal del Canal 7. Al poco tiempo nació el Canal 4. Ambos canales televisivos privados (4 y 5) se lanzaron a una competencia duopólica por la preferencia del público que duró cerca de veinte años, hasta inicios de los años ochenta.

En 1971 el gobierno militar de entonces creó el Instituto Nacional de Teleducación (INTE), con el objetivo de que produjera programas educativos para la televisión. En ese mismo año, dispuso la estatización del $51 \%$ de las acciones de los canales de televisión, y del 25\% de las acciones de las principales radios. En 1972 el Ministerio de Educación creó la Dirección de Comunicación Colectiva con la finalidad de "establecer las normas referentes al contenido de los programas de radiodifusión y publicidad". Se normaron los contenidos de la publicidad y los programas de radio y televisión, y a fines de ese mismo año, aprobó el acuerdo relativo a la Organización Internacional de Telecomunicaciones por Satélite, el mismo que había sido suscrito en Washington en 1971.

En 1976, en pleno gobierno militar y con financiamiento estatal, los Canales 4 y 5 adoptaron el formato de televisión a color, luego de

1 Diario El Comercio. (2000). “El Siglo XX de El Comercio 1920-1929”. Lima, Plaza Janés, p. 108. 
más de veinte años de su invención (1954). En 1980, año en que se restableció la democracia, el gobierno devolvió los canales de televisión, radios y diarios a la plena propiedad de sus accionistas y desactivó todos los mecanismos de regulación de contenidos; al mismo tiempo desactivó el proceso de reforma de la educación del país. En la década de los ochenta irrumpieron nuevos canales hasta copar la banda VHF (canales del 2 al 13) e iniciar la exploración de la banda UHF.

Durante toda la historia de la televisión peruana, la televisión estatal siempre tuvo un papel discreto, con niveles de sintonía que no llegaban al $2 \%$ nacional. Por esta causa no resultó traumático que a fines de los ochenta el Canal 13, de naturaleza educativa estatal y que venía siendo operado por la Universidad de Lima, fuera también privatizado por el gobierno de entonces. En el año 2000 ya no había señales UHF disponibles en la ciudad de Lima.

Desde los años ochenta en que desaparecen los elementos regulatorios estatales, la televisión peruana pasó a dedicarse por completo al entretenimiento. Sus contenidos fueron y son mayormente telenovelas latinoamericanas y películas norteamericanas. La producción televisiva nacional se centra en los informativos diarios, en los deportes, en el humor, y últimamente en los comentarios sobre la vida privada de la gente pública. Esta perspectiva del negocio televisivo sigue vigente hasta la fecha como si fuera un mandamiento del modelo de la sociedad peruana. Hay que mencionar que el negocio de la publicidad peruana llega actualmente a los mil millones de dólares anuales, de los cuales un $80 \%$ es captado por la televisión.

Del mismo modo que en el caso de la radio, el Perú adoptó el modelo norteamericano del negocio del broadcasting, es decir autofinanciado, donde ni el Estado ni la sociedad cuentan con mecanismos regulatorios sobre los contenidos emitidos por las televisoras, a diferencia del buen modelo alemán, por ejemplo, donde el estado financia parte de los costos operativos de las señales de radio y televisión, por lo cual exige y mantiene un mínimo de calidad cultural y educativa en su programación.

En el Perú, si algún líder o institución sugiere alguna iniciativa para orientar o regular contenidos televisivos, inmediatamente los broadcasters lo denuncian como agresión a la libertad de prensa, y reclaman autonomía absoluta. De facto, ellos se han auto-asignado el rol de constituir un nuevo poder del Estado compitiendo con el poder judicial. La arrogación de este poder ha conducido a una inédita forma de apropiación de las señales del espectro electromagnético que el gobierno, a nombre de la nación, ha asignado condicionalmente a estas empresas para operar sus estaciones.

\section{Los satélites}

En 1957 Rusia lanzó el primer satélite artificial. Desde entonces se calcula que unos 6500 satélites han sido lanzados por los países desarrollados y que unos 3000 de ellos se mantienen en órbita. La mayoría de los mismos se puede clasificar en dos grupos: satélites de comunicaciones y satélites uso militar. Sobre los primeros sabemos mucho, pero sobre los segundos casi nada.

Sabemos por ejemplo que los satélites de comunicaciones son de tres tipos: pequeños o de órbita baja (300 km de altitud), medianos (4000 $\mathrm{km})$ y geoestacionarios o de órbita alta (37000 $\mathrm{km})$. Estos últimos pueden tener el peso de un microbús y ser capaces de reflejar unos 1000 canales de televisión al mismo tiempo o conectar varios cientos de miles de teléfonos.

Los satélites podrían servir al Perú para crear redes de educación nacional, redes de salud públi$\mathrm{ca}$, redes de seguridad nacional, redes para controlar los cardúmenes y la pesca en el mar territorial, redes para advertir el daño al medio ambiente, particularmente el causado por los narcotraficantes. El ministro del Ambiente, Dr. Antonio Brack Egg, ha anunciado hace tiempo que solamente en el departamento de San Martín tenemos más de cinco millones de hectáreas de selva depredadas; sin embargo el Perú, a pesar de ser un país de una extensión de 1.2 millones de $\mathrm{km}^{2}$, no tiene un solo satélite. Se podrá argumentar que su costo es prohibitivo, pero no es así. Un satélite mediano puede costar alrededor de 30 millones de dólares, con una vida útil promedio de quince años. El porqué el Perú no se lanzado a adquirir e iniciar el manejo de esta tecnología es un verdadero misterio. Países como Brasil, México y la misma Argentina tienen redes satelitales. Los científicos chilenos desde hace más de una década vienen trabajando con sus pares británicos. Probablemente Chile lance en el año 2008 una flotilla satelital.

No es que los peruanos hayan ignorado la tecnología satelital. Su uso comenzó en los años 
ochenta cuando la televisión privada comenzó a transmitir programas internacionales, sobre todo deportivos, apoyándose en la tecnología del Panamsat, una cadena de satélites medianos de propiedad de una compañía norteamericana. Posteriormente, en los años noventa, la tecnología satelital comenzó a ser utilizada para la conformación de redes radiales satelitales con sede matriz en Lima. Así nacieron las cadenas RPP (noticiosa), radio Panamericana, radio Mar, radio Ritmo, etc.

Es decir que, mientras que los países desarrollados aplicaron la tecnología satelital inmediatamente para crear redes de escuelas, de hospitales, farmacias, comisarías y de sus sistemas de sistema de defensa nacional, el Perú la aplicó casi exclusivamente para transmitir programas de entretenimiento en la televisión y en la radio.

Ejemplo patético de nuestro atraso tecnológico y de la falta de previsión del gobierno en este tema ha sido el nulo servicio de telecomunicaciones para mitigar el terremoto de Pisco en el año 2007. Tener totalmente operativo un sistema de telecomunicaciones para emergencias nacionales (vía radial, satelital, microondas, etc.) es de responsabilidad exclusiva de cualquier Estado, y no de las empresas operadoras privadas de telefonía. Ojalá que ese ejemplo sirva de lección para el propio Perú así como para otros estados pequeños alrededor del mundo, y que permita que las fuerzas armadas peruanas entiendan la importancia de contar con un sistema de comunicaciones funcional. ¿Puede alguien imaginarse el nivel absolutamente desventajoso del Perú en un escenario de guerra con otro país, con este estado de atraso en las telecomunicaciones?

\section{La Videograbadora}

En la década del noventa llegó la videograbadora y la filmadora. Cada biblioteca pública de los países desarrollados fue llenada de videos educativos sobre idiomas, técnicas, oficios, etc., hasta clases y cursos completos sobre diferentes asignaturas como Geometría, Biología, Química o Física. En Canadá y otros países se construyeron aulas de clases con sistema de filmación incluidos para que cada clase pudiera ser vista en diferido por aquellos alumnos distantes o que por razones de mal clima no hubieran podido asistir a clases.

En el Perú de los noventa, cada colegio adquirió una filmadora con su correspondiente video-reproductora. Eso fue bueno pues ¿fue para mejorar la educación?, ¿tal vez para enseñar primeros auxilios en casos de desastres? iNo! Fue para el registro de las ceremonias y de todos los actos sociales de los directores o de las APAFAS. Es decir, para asuntos sentimentales privados, para la superficialidad, para el entretenimiento, pero no se usó para los desafíos educativos.

\section{La Telefonía}

Este invento de Alexander Graham Bell creado en 1876 tuvo un lentísimo ingreso al Perú y muy poco ha sido explotado para promover el desarrollo nacional. El teléfono fue una herramienta de las empresas y un privilegio de ciertas oficinas de gobierno. Por décadas la telefonía estuvo gestionada por empresas de naturaleza estatal o cuasi estatal, con una inversión débil en infraestructura que hizo que poseer un aparato en el hogar fuera un signo de estatus de las familias más pudientes.

Ciertamente que desde los años sesenta se ha usado para complementar la red de telegrafía nacional, pero nunca se le usó, por ejemplo, para diseñar e implantar un sistema educativo rural a distancia, tal como se ha utilizado en Canadá, país con cierta semejanza por su extensión y difícil acceso a las zonas rurales. Tampoco ha sido diseñado ni usado para crear una red de salud pública rural, o incluso de vigilancia epidemiológica. Tampoco ha sido utilizada para promover la mejora de las técnicas de agricultura, de ganadería o de artesanía.

Pero, a diferencia de los medios de telecomunicación anteriores, la evolución del sector telefonía en el Perú resulta actualmente muy auspiciosa. Las cifras de la evolución de la posesión telefónica son asombrosas (ver Figura № 1). En 1994 , fecha en que se privatizaron los servicios telefónicos del Perú, el país tenía una densidad telefónica de 3.4 teléfonos por cada cien habitantes; seis años más tarde (en el 2000), Perú ya tenía 12 teléfonos y luego 7 años más tarde llegábamos a los 65 . Es decir un crecimiento del $1,700 \%$ en 13 años. Esto genera mucha ilusión si lo comparamos con las densidades de los Estados Unidos: lo que nosotros teníamos en 1994 ellos lo lograron en 1901, es decir teníamos un atraso relativo de 91 años; pero trece años más tarde llegamos a tener 65 teléfonos, cifra que corresponde al año 2000 para el país del norte; esto quiere decir que el atraso relativo se redujo a solamente siete años. 
Figura №1. Evolución de la densidad telefónica peruana comparada con la de los Estados Unidos de América

\begin{tabular}{|c|c|c|c|}
\hline Año & $\begin{array}{c}\text { Densidad Telefónica (*) } \\
\text { Perú }\end{array}$ & $\begin{array}{c}\text { Año correspondiente en } \\
\text { Estados Unidos (**) }\end{array}$ & Diferencia en años \\
\hline 1994 & 3 & 1903 & 91 años \\
\hline 2000 & 12 & 1930 & 70 años \\
\hline 2007 & 65 & 2000 & 7 años \\
\hline
\end{tabular}

(*) Densidad Telefónica en el número de teléfonos por cada 100 habitantes de un país. Cifras obtenidas de la web de OSIPTEL.

(**) Cifras obtenidas de la web de la FCC (Federal Communications Comission).

Hay que indicar que las cifras de evolución de otros países latinoamericanos son aún más espectaculares que las peruanas.

No obstante eso, existe la expectativa deque el crecimiento geométrico de la telefonía, que coincide con un ciclo económico expansivo del Perú, contribuya al crecimiento del PBI y al combate contra la pobreza, tal como ha sido demostrado en otros países emergentes como la República Popular China. Particularmente hay gran expectativa por su impacto sobre el comercio electrónico, a través del alquiler de líneas dedicadas o de banda ancha.

Hay amplia expectativa sobre su uso en la construcción de redes inter-empresariales tipo cluster o negocio a negocio (B2B), negocio a cliente (B2C) y gobierno a negocio (G2B); así como en la construcción de redes con proveedores y clientes públicos y privados de los tipos supply chain management (SCM) y customer relationship management (CRM), gobierno a cliente $(\mathrm{G} 2 \mathrm{C}) \mathrm{y}$ cliente a cliente (C2C).

\section{La Computadora, Internet, Youtube}

A inicios de los noventa llegó el correo electrónico e Internet al Perú. Varias instituciones y personalidades se entusiasmaron con este acontecimiento como el Radio Club Peruano; por su parte, las universidades conformaron una entidad tipo cooperativa llamada Red Científica Peruana (RCP). Sin embargo, la falta de visión de las propias autoridades universitarias y la carencia del apoyo del Estado hizo que este proyecto fracasara. La idea era constituir una red de ciencia y educación nacionales con contactos internacionales que solo quedó en una buena intención.

Finalmente, a fines de los noventa e inicios del 2000 llegó internet. En el Perú se han estableci- do tres grandes usuarios de servicios de computación y de Internet: el más numeroso lo conforman los hogares, luego las empresas y, finalmente, el gobierno. La penetración de las computadoras en los hogares se calcula que está por el orden del $15 \%$ pero el acceso de los ciudadanos y de los jóvenes a este servicio es mucho mayor, debido a que la pequeña empresa privada construyó un modelo de empresa llamada "cabinas públicas" de amplia difusión en todo el país.

En el año 2001 el gobierno del Presidente Toledo lanzó un publicitado proyecto informático llamado "Proyecto Huascarán" para difundir el Internet hasta los lugares menos accesibles y a los escolares más vulnerables; inclusive el presidente logró una simbólica entrevista con el presidente de Microsoft, Bill Gates. Sin embargo, después de todo el despliegue no se conocen mayores resultados de dicho proyecto. Parece que todo resultó ser una propaganda política del gobierno. Mas bien, quienes cumplieron la tarea de extender el servicio de Internet hasta los lugares más alejados del país fueron las MYPES privadas con la difusión de sus cabinas públicas en los distritos donde existe la red telefónica. Sin embargo, cabe preguntar ¿cuál es el uso que el público en general da a internet? La respuesta se puede dar en cuatro palabras: e-mail, chat, juegos y búsquedas esporádicas.

Como reacción a la pésima calificación que los organismos educativos internacionales han dado a le educación pública peruana que ocupó uno de los últimos lugares en comprensión de lectura y de operaciones matemáticas, a comienzos del año 2008 el gobierno del presidente Alan García ha decidido obsequiar cientos de miles de laptops a los escolares de los colegios rurales de la sierra. Todo parece indicar, sin embargo, que paralelamente no existe un plan de uso de las mis- 
mas. Por ejemplo, no se conoce que previamente se haya construido un banco de datos de contenidos escolares, tampoco se conoce que se haya puesto en marcha algún plan de entrenamiento a los maestros. Consecuentemente, si no hay contenidos escolares disponibles de tipo mandatario, el uso que los niños van a dar a las laptops va a ser el mismo que se da en las cabinas públicas: mail, chat, juegos y búsquedas sin asesoramiento, cuando el Internet escolar podría convertirse en un vehículo de socialización de la juventud (e-mail y chat) y de entretenimiento (toda clase de webs y blogs desde deportivos hasta de hardcore sex).

En la educación escolar corremos el riesgo de dejar pasar la mejor oportunidad tecnológica para superar el atraso e ingresar plenamente a la modernidad. Lo mismo ocurre en la educación superior: aunque estamos en pleno siglo XXI son pocas las universidades públicas que utilizan la tecnología de e-commerce (EDI) inventada hace veinte años. Aún no existe una red universitaria que tenga una sola base de matrículas, registros, notas e investigaciones, y se puede afirmar -sin temor a equivocación- que en cuanto a organización estamos como las universidades norteamericanas de los años sesenta. Cada universidad, por su cuenta, hace lo que puede con esta tecnología y los profesores y alumnos -también por su cuenta- están buscando beneficiarse de lo que otras universidades están ofreciendo a través del Internet (caso del Open Courseware del Instituto Tecnológico de Massachussets) o de los servicios complementarios como Youtube o I-tunes.

\section{Conclusión y Discusión}

La historia de la penetración de los medios de comunicación electrónica en el Perú refleja que la tecnología de comunicaciones entusiasma a los peruanos, nos gusta y es acogida esencialmente para la diversión y el entretenimiento. Los actores políticos, académicos, sociales, y los simples ciudadanos han mirado la tecnología de comunicaciones desde la óptica de la diversión, del entretenimiento. En esto existe una marcada diferencia con los países desarrollados, donde cada invento ha tenido una aplicación planificada para el desarrollo económico y social, sea por el gobierno, por las empresas o por las universidades, es decir por los actores del desarrollo.

Quiero mencionar que en 1972 el Ministry of International Trade and Industry (MITI) de Japón formuló un conjunto de leyes para que todas las empresas industriales adoptaran el uso de la computadora en la fabricación, bajo el lema "El chip, el arroz de la industria". El resultado es que diez años después Japón se volvió el país con el mayor nivel de automatización del mundo. En el Perú, hasta la fecha, nadie es responsable en los hechos de las aplicaciones informáticas. La informática crece sin orden ni concierto.

Con todos los antecedentes descritos podemos afirmar que no va a darse ningún proceso espontáneo de innovación tecnológica en el Perú; por el contrario, parece necesaria la construcción urgente de un organismo promotor, regulador y directriz de la innovación tecnológica en el país, con capacidades decisorias, tal como lo tienen otros países como Chile, por ejemplo. Tal vez es necesaria la recreación de una oficina de patentes que comience recogiendo las funciones del finado Instituto Nacional de Normas Técnicas (INTINTEC) y que trabaje en armonía con el sector empresarial, con el sector público, así como con las universidades. Este organismo debería evaluar el atraso tecnológico del país y realizar un inventario de las tecnologías disponibles, para finalmente aprobar y poner en marcha el plan estratégico tecnológico de largo plazo del país, con su financiamiento correspondiente.

Si el Perú toma conciencia de esta realidad y actúa rápidamente habrá avanzado un poquito en el camino hacia el desarrollo, además de empezar a hacer bien las cosas. 


\section{BIBLIOGRAFÍA}

Bonilla, Heraclio. (1972). Informes de los cónsules británicos. Tomo I. Lima, Instituto de Estudios Peruanos (IEP), p. 301.

Cuánto Publicaciones. (2007). Perú en Números 2007. Lima, Cuánto Publicaciones.

Gibson, Stephen W. (1987). Cellular Mobile Radiotelephones. Englewood Cliff, Prentice Hall.

Graham, John F. (1995). Space. Estados Unidos, NASA: En sitio web: www.space.edu

Gross, Lynne Schafer. (1983). Telecommunications. An introduction to Radio, Television and other Electronic Media. Segunda Edición. Iowa, Wcb. Dubuque.

Oslin, George P. (1992). The Story of Telecommunications. Macon, Mercer University Press.

\section{PÁGINAS WEB}

http://inventors.about.com/science/inventors/library/ inventors

http://www.cia.gov

http://www.discovery.com/stories

http://www.fcc.gov

http://www.fht-esslingen.de/telehistory

http://www.hispasat.com

http://www.intelsat.com

http://www.loc.gov

http://www.mediahistory.com

http://www.nasa.gov

http://www.osiptel.gob.pe

http://www.space.edu

http://www.telefonica.com.pe

http://www.sciencemadesimple.com

http://www.wikipedia.com 